\title{
The Effect of Pulse Amplitudes on Quality of Determining Distribution of Pulses Forcing Vibration of an Damped Oscillator
}

\author{
A. OzGA* \\ AGH University of Science and Technology, Faculty of Mechanic Engineering and Robotics, \\ Department of Mechanics and Vibroacoustics, Al. Mickiewicza 30, 30-059 Krakow, Poland
}

\begin{abstract}
The paper presents another phase of the study aimed at determining distributions of random pulses forcing vibration of an oscillator with damping. At this stage, the impact of the pulses amplitudes on distributions determined in a finite time interval is discussed. Application of a mathematical model in simulations allows to determine the differences between the distributions generated in MATLAB environment and those determined by a function. The experiment was designed so that the qualitative analysis of the issue was possible.
\end{abstract}

DOI: 10.12693/APhysPolA.128.A-67

PACS: $45.05 .+\mathrm{x}, 45.30 .+\mathrm{s}, 45.90 .+\mathrm{t}$

\section{Introduction}

The paper reports a subsequent stage of research on the problem which can be defined generally as an attempt to determine distributions of random pulses forcing vibration of an oscillator with damping [1]. The study started with construction of a mathematical model [2] and further, some research methods were developed [3] which allowed to find the distribution of amplitudes of vibration forcing pulses in spite of numerical errors [4] or for the time interval subjected to analysis being too short. The problem was also considered specifically in context of the pulse rate [5] and parameters of the vibrating system [6]. Both qualitative and quantitative methods were used while approaching the issue [7].

In this stage of the research project outlined above, the objective consists in carrying out an analysis of a vibrating system from the point of view of the number of pulses making up a distribution forcing the vibration and values of their stochastic amplitudes. The analysis is expected to provide answers to the following two questions:

1. What are the differences in the determined distributions if vibration are forced by distributions of pulses with similar values of amplitudes?

2. How will the systems with different parameters $b$ and $c$ behave when vibration are forced by distributions of pulses with amplitude values differing significantly and what will be the effect of such significant differences on the computed distributions?

When applied to vibroacoustics, qualitative methods have certain limitations in interpretation of results [8] as regards construction of mathematical models $[9,10]$, simulations [11, 12], or experiments [13, 14, 15]. However, on the grounds of both the literature quoted above and

*e-mail: aozga@agh.edu.pl the study presented in the present paper it is possible to claim that analyzing a few variables at a time and asking precise questions [16] represent an effective approach to specific problems of vibroacoustics $[17,18]$.

\section{The method}

Simulations connected with the analysis of systems with vibration forced by random series of pulses represented by a function $f(t)$ are a complex issue due to the intrinsic nature of the problem [19]. A mathematical model proposed in [20] allows to determine the distribution of pulses occurring over an infinite period of time $(t \rightarrow \infty)$ and forcing motion of a one-dimensional damped oscillator described by equation

$$
x^{\prime \prime}(t)+2 b x^{\prime}(t)+a^{2} x(t)=\sum_{t_{i}<t} \eta_{i} \delta\left(t-t_{i}\right)
$$

with initial conditions $x(0)=0$ and $x /(0)=0$, where $x$ is the deflection of the system from its equilibrium position, the damping coefficient $b$ and the frequency $c=\left(a^{2}+b^{2}\right)^{1 / 2}$ are parameters of the vibrating system, $i=1,2,3, \ldots$ is the pulse number, $\eta_{i}$ are random amplitudes of pulses, and $t_{i}$ are random instants of time at which the pulses occur. It is further assumed that the time intervals $t_{i}-t_{i-1}$ between subsequent pulses have the exponential distribution with the pulse rate $\lambda$, $\eta_{i}$ form a sequence of independent identically distributed random variables with a finite expectation value, and the set of values $\left\{\eta_{1}, \eta_{2}, \ldots . \eta_{k}\right\}$ is finite with probabilities $p_{i}=p\left(\eta_{i}\right)$.

Taking into account the fact that in all papers making up the research project of which this study is a part, vibration of the system were simulated by employing electromechanical analogies, it has been assumed that the position $x$ of the system is a dimensionless quantity, which involves corresponding selection of units for oscillator parameters $a$ and $b$ as well as for pulse amplitude values $\eta_{i}$ and the pulse rate $\lambda$. And so, the parameters of the oscillator were assumed to be $b=8281 \mathrm{~s}^{-1}$ 
and $c=300180 \mathrm{~s}^{-1}$, which corresponded to the analogous RLC circuit with inductivity $L=5 \mathrm{mH}$, capacity $C=0.2 \mathrm{nF}$, and a voltage source [18]. On that side of the analogy, system position is obviously given in volts.

The values of stochastic moments $m_{n}$ determined from $x(t)[2]$ are put in the following equations in order to determine the distributions $\bar{p}_{i}$ :

$$
\begin{aligned}
& \sum_{i=1}^{k} \bar{p}_{i}\left(\left(m_{i}(t) m_{1}(t)-m_{i+1}\right) \eta_{i}\right. \\
& \left.\quad+\sum_{j=1}^{n}\left(\begin{array}{l}
n \\
j
\end{array}\right) m_{n-j}(t) m_{1}(t) \eta_{i}^{j+1} \frac{C(j+1)}{C(1) c^{j}}\right)=0, \\
& \sum_{i=1}^{k} \bar{p}_{i}=1
\end{aligned}
$$

where $k$ is the number of the sought values of random amplitudes $\eta_{k}$ for $i=1,2, \ldots, k-1, m_{i}(t)$ is the $i$-th stochastic moment of the random variable $x$, and for $j>1$ and even $j$

$$
C(j)=\frac{j !}{\prod_{r=0}^{j / 2-1}\left((j b / c)^{2}+(2 r)^{2}\right)} \frac{c}{j b},
$$

whereas for odd $j>0$,

$$
C(j)=\frac{j !}{\prod_{r=0}^{(j-1) / 2-1}\left((j b / c)^{2}+(2 r+1)^{2}\right)} .
$$

In simulations carried out in earlier papers [2, 4, 5], distributions $\bar{p}_{i}$ were determined with the use of Eqs. $(2-5)$ for the pseudorandom variable $\eta_{i}$ assuming three values: $\eta_{1}=845778.47 \mathrm{~s}^{-1}, \eta_{2}=\eta_{1} / 2$, and $\eta_{3}=\eta_{1} / 10$.

Using high-performance computers and the PLgrid platform it was possible to calculate the distributions for six values of $\eta_{i}$. The conclusions drawn on the basis of a former study [6] allowed to design the experiment so that a qualitative analysis of the presented results become possible.

In the simulations, the following algorithm is adopted: firstly, a pseudorandom function rand () defining a uniform distribution in the interval $(0,1)$ is used to obtain random time intervals between pulses

$$
\operatorname{rand}_{t}=-\frac{1}{\lambda} \ln (1-\mathrm{rand}) \text {. }
$$

At the subsequent step, random amplitude values $\eta_{i}$ are derived corresponding to the imposed probability $p_{i}$.

No matter what pulse rate $\lambda$ is selected for a finite time interval, determination of the imposed distribution $p_{i}$ (e.g. $p_{i}=1 / 3$ ) is impossible. Therefore, occurrences of each $\eta_{i}$ are counted in the simulations. The sum of occurrences of a given pulse divided by the sum of occurrences of all pulses gives the distributions that will be denoted by $\tilde{p}_{i}$ in the following.

In all qualitative investigations described below, the difficulties encountered in determining a series of pulses forcing an oscillator are analyzed by computing the difference between the distributions $\left|\bar{p}_{i}-\tilde{p}_{i}\right|$. Earlier simulations $[2,6]$ show that the differences between distributions generated in MATLAB environment $\tilde{p}_{i}$ and the distributions $\bar{p}_{i}$ which were determined on the basis of the function representing deflection of the system are influenced by: the parameters $b$ and $c$ of the oscillator, pulse rate $\lambda$, length of the analyzed time interval, and the instant of time at which the difference is being determined. In the applied algorithm, the difference is determined with the step of $10^{6}$, at every second. A single series of vibration is analyzed for $t$ ranging from 0 to $7200 \mathrm{~s}$.

\section{An analysis of systems with slightly differing vibration-forcing pulse amplitudes}

At the first stage of the analysis, three distributions $\Phi_{i}(i=1,2,3)$ were compared, for which the pulses amplitudes forcing vibration differed slightly and values of the remaining parameters $b, c, \lambda$ influencing the analyzed function were the same. And so, for the purpose of simulations, the value $\eta_{1}=845778 \mathrm{~s}^{-1}$ was adopted and three distributions with occurrence of each pulse imposed with probability $p_{i}=1 / 6$ and pulse amplitudes assuming six different values:

$$
\begin{aligned}
& \text { 1. } \Phi_{1}: \eta_{1}, \eta_{2}=0.9 \eta_{1}, \eta_{3}=0.6 \eta_{1}, \eta_{4}=0.5 \eta_{1} \text {, } \\
& \eta_{5}=0.3 \eta_{1}, \eta_{6}=0.1 \eta_{1} \\
& \text { 2. } \Phi_{2}: \eta_{1}, \eta_{2}=5 \eta_{1} / 6, \eta_{3}=4 \eta_{1} / 6, \eta_{4}=3 \eta_{1} / 6 \text {, } \\
& \eta_{5}=2 \eta_{1} / 6, \eta_{6}=\eta_{1} / 6 \\
& \text { 3. } \Phi_{3}: \eta_{1}, \eta_{2}=0.9 \eta_{1}, \eta_{3}=0.5 \eta_{1}, \eta_{4}=0.4 \eta_{1} \text {, } \\
& \eta_{5}=0.3 \eta_{1}, \eta_{6}=0.1 \eta_{1} \text {. }
\end{aligned}
$$

As it can be seen, the values of amplitudes are selected so that the strongest pulse occurring in all distributions is $\eta_{1}$. In the distribution $\Phi_{2}$, amplitudes $\eta_{i}$ for $i>1$ assume values being multiples of $1 / 6 \eta_{1}$. The other two distributions are not evenly spaced and assume the values between $\eta_{1}$ and $\eta_{1} / 10$. In distributions $\Phi_{1}$ and $\Phi_{3}$, five pulses have the same amplitude values. The difference between the distributions is determined by the pulse with the amplitude value $0.6 \eta_{1}$ occurring in $\Phi_{1}$ and the pulse with the amplitude value $0.4 \eta_{1}$ occurring in $\Phi_{3}$.

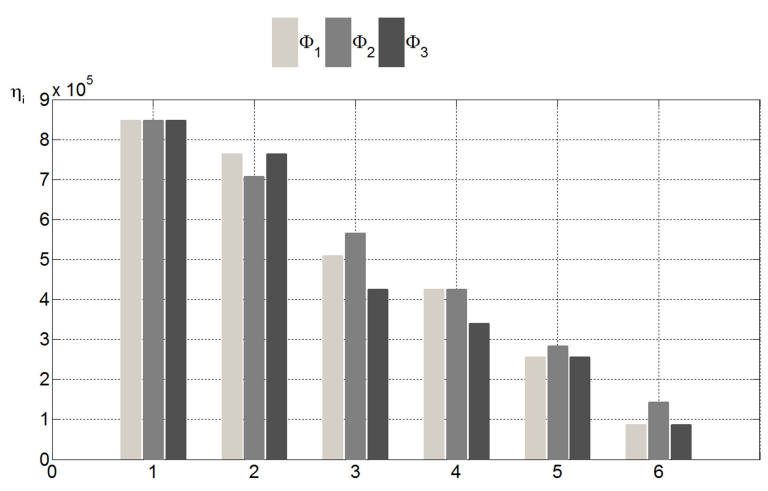

Fig. 1. The applied method of combining of pulses.

In each of the analyzed distributions, pulses with different amplitudes $\eta_{i}$ occur at the same random moment 
of time $t_{i}$, whereas an interdependence is maintained between the distributions such that if the pulse of the highest amplitude $\eta_{1}$ occurs in the first distribution, the second and the third distribution also receive the hits of the strongest pulse. The way in which particular groups of pulses are interrelated is shown in Fig. 1. Thanks to the applied comparative analysis, it is possible to examine what is the effect of amplitude values $\eta_{i}$ on differences $\left|\bar{p}_{i}-\tilde{p}_{i}\right|$ (Fig. 2).
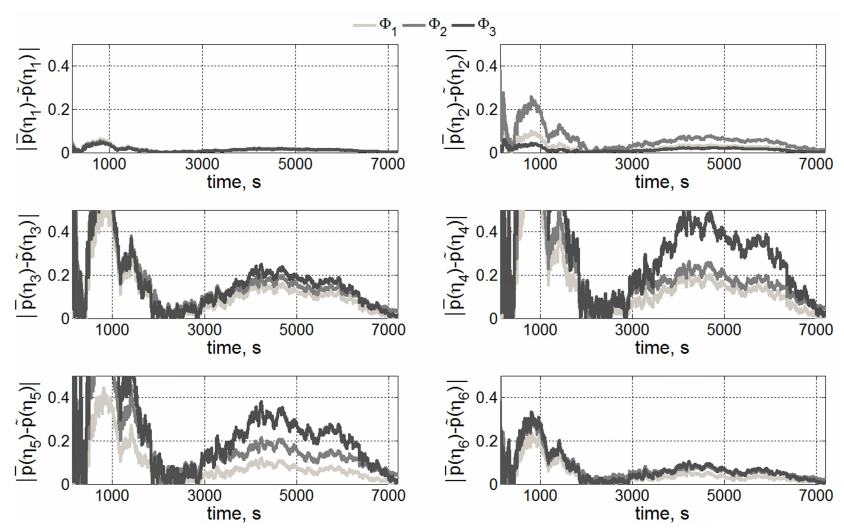

Fig. 2. Differences $\left|\bar{p}_{i}-\tilde{p}_{i}\right|$ determined for distributions $\Phi_{1}, \Phi_{2}$, and $\Phi_{3}, \lambda=500 \mathrm{~s}^{-1}, b=8281 \mathrm{~s}^{-1}$, $c=300180 \mathrm{~s}^{-1}$, and $\eta_{1}=845778 \mathrm{~s}^{-1}$.

The qualitative method applied in the research allows to conclude that even small differences between amplitudes of vibration-forcing pulses result in occurrence of differences between distributions determined from the function representing observed vibration of the system.

The diagrams shown in Fig. 2 confirm the observation from the previous research [19] that the distribution of the strongest pulse $\eta_{1}$ is determined with the smallest error. It cannot be confirmed, however, that the distribution of the weakest pulse $\eta_{6}$ is determined with the largest error, no matter whether the values of the sought amplitudes are evenly $\left(\Phi_{2}\right)$ or unevenly $\left(\Phi_{1}\right.$ and $\Phi_{3}$ ) distributed.

An analysis of a single function representing vibration of the system shows that for six pulses with given amplitudes within a finite time interval from 0 to $7200 \mathrm{~s}$, the distributions were determined with an error too large to claim that the use of the method in technological applications would be justified [1].

\section{An analysis of systems forced with pulses with significantly differing amplitudes}

To confirm that minor differences in pulse amplitudes influence the errors in the determined distributions, it was sufficient to present just one such case subjected to qualitative studies, and this was done in the previous section. However, if the analysis concerns differences $\left|\bar{p}_{i}-\tilde{p}_{i}\right|$ that occur as a result of significant differences between pulse amplitudes, the experience acquired in the previous studies [6] shows that this case should be considered in the context of oscillators characterized with different parameters or, in other words, for different oscillators.
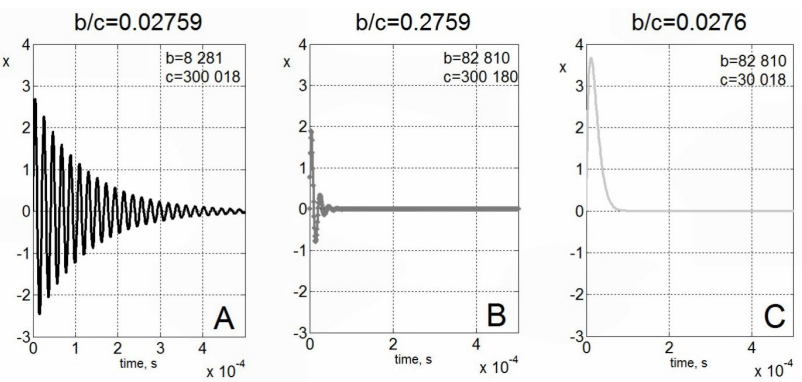

Fig. 3. Vibration forced by a pulse with amplitude $\eta_{1}=845778 \mathrm{~s}^{-1}$ in three oscillators with parameters $b$ and $c$ given in the diagrams.

Simulations were conducted for three damped oscillators (Fig. 3) labeled with letters A, B, and C. The parameters of oscillators $\mathrm{B}$ and $\mathrm{C}$ were selected bearing in mind specific needs of the simulation.

In this part of the considerations, simulations will be conducted for another three distributions denoted by $\Phi_{4}$, $\Phi_{5}$, and $\Phi_{6}$ :

1. $\Phi_{4}: p\left(\eta_{1}\right)=1 / 3, p\left(\eta_{2}\right)=1 / 3, p\left(\eta_{3}\right)=1 / 3$, $p\left(\eta_{4}\right)=0, p\left(\eta_{5}\right)=0$, and $p\left(\eta_{6}\right)=0 ;$

2. $\Phi_{5}: p\left(\eta_{1}\right)=0, p\left(\eta_{2}\right)=0, p\left(\eta_{3}\right)=1 / 3, p\left(\eta_{4}\right)=1 / 3$, $p\left(\eta_{5}\right)=1 / 3$, and $p\left(\eta_{6}\right)=0$;

3. $\Phi_{6}: p\left(\eta_{1}\right)=0, p\left(\eta_{2}\right)=0, p\left(\eta_{3}\right)=0, p\left(\eta_{4}\right)=1 / 3$, $p\left(\eta_{5}\right)=1 / 3$, and $p\left(\eta_{6}\right)=1 / 3$.

for which the variable $\eta_{i}$ assumes six values:

$$
\begin{aligned}
& \eta_{1}, \eta_{2}=5 \eta_{1} / 6, \eta_{3}=4 \eta_{1} / 6, \eta_{4}=3 \eta_{1} / 6, \eta_{5}=2 \eta_{1} / 6, \\
& \eta_{6}=\eta_{1} / 6 .
\end{aligned}
$$

The distributions were selected so that in $\Phi_{4}$, vibration are forced by the three strongest pulses, i.e. $\eta_{1}$, $\eta_{2}$, and $\eta_{3}$; in $\Phi_{5}$, vibration are forced by pulses $\eta_{3}, \eta_{4}$, and $\eta_{5}$; and in distribution $\Phi_{6}$, vibration are forced by the three weakest pulses, i.e. $\eta_{4}, \eta_{5}$, and $\eta_{6}$.

In order to assess the effect which numerical errors issuing from the form of the Eqs. (2-6) have on the difference $\left|\bar{p}_{i}-\tilde{p}_{i}\right|$, the distributions are determined for either six or three values of $\eta_{i}$. The obtained results are presented in Figs. 4-9.

Comparing the results presented in Figs. 4, 6 and 8 it can be seen that the strongest pulses are responsible for occurrence of largest values of the difference $\left|\bar{p}_{i}-\tilde{p}_{i}\right|$. If only distribution $\Phi_{4}$ is taken into account, analysis of a single waveform shows that within a finite time interval from 0 to $7200 \mathrm{~s}$ and for six values of amplitudes $\eta_{i}$, the distributions were determined with an error too large for the method to be acceptable in technological solutions. However, when the vibration are analyzed for three values of amplitudes $\eta_{i}$ only (Fig. 5), the effect of numerical errors on the difference between distributions is much less remarkable. 

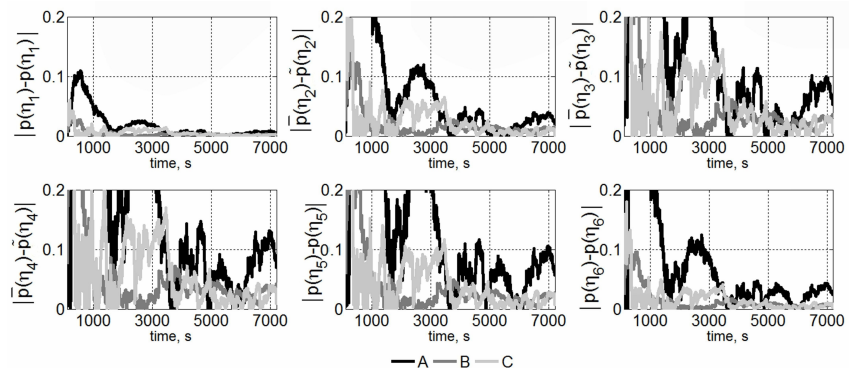

Fig. 4. Differences $\left|\bar{p}_{i}-\tilde{p}_{i}\right|$ determined for the distribution $\Phi_{4}, \lambda=1000 \mathrm{~s}^{-1}$, and six pulse amplitude values.
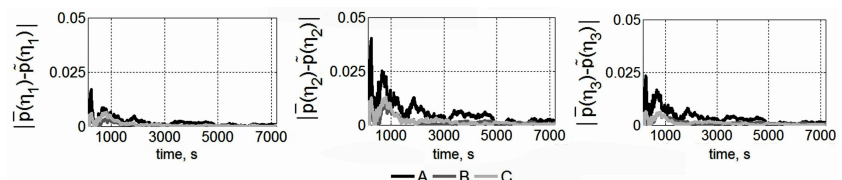

Fig. 5. Differences $\left|\bar{p}_{i}-\tilde{p}_{i}\right|$ determined for the distribution $\Phi_{4}, \lambda=1000 \mathrm{~s}^{-1}$, and three pulse amplitude values.
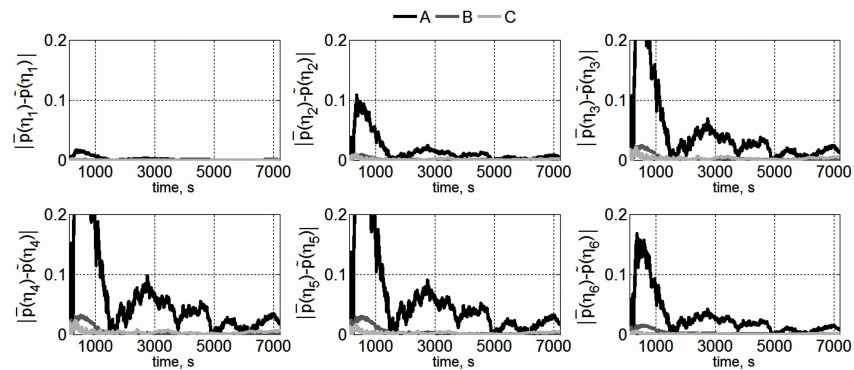

Fig. 6. Differences $\left|\bar{p}_{i}-\tilde{p}_{i}\right|$ determined for the distribution $\Phi_{5}, \lambda=1000 \mathrm{~s}^{-1}$, and six pulse amplitude values.
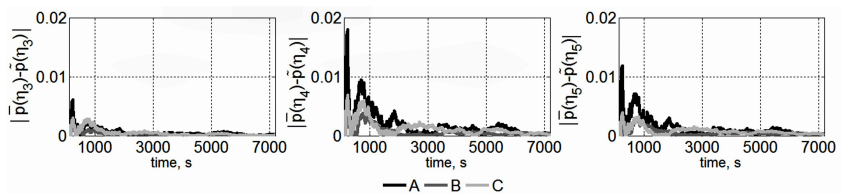

Fig. 7. Differences $\left|\bar{p}_{i}-\tilde{p}_{i}\right|$ determined for the distribution $\Phi_{5}, \lambda=1000 \mathrm{~s}^{-1}$, and three pulse amplitude values.
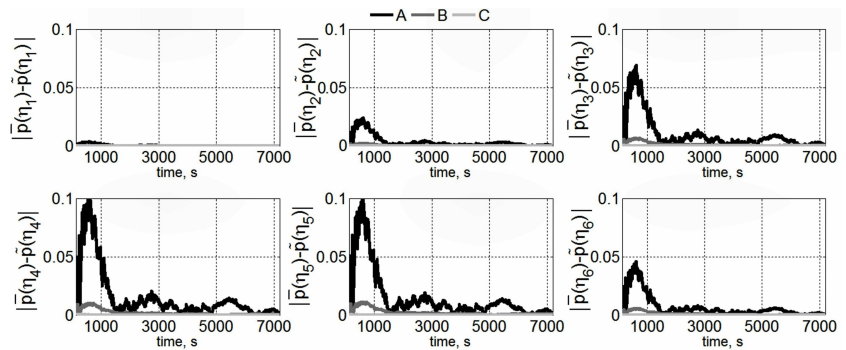

Fig. 8. Differences $\left|\bar{p}_{i}-\tilde{p}_{i}\right|$ determined for the distribution $\Phi_{6}, \lambda=1000 \mathrm{~s}^{-1}$, and six pulse amplitude values.

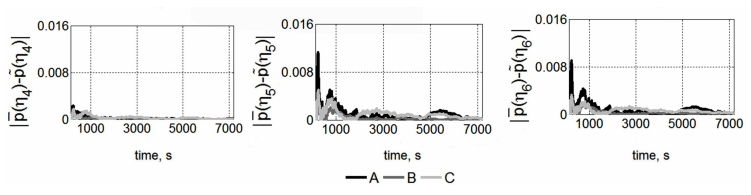

Fig. 9. Differences $\left|\bar{p}_{i}-\tilde{p}_{i}\right|$ determined for the distribution $\Phi_{5}, \lambda=1000 \mathrm{~s}^{-1}$, and three pulse amplitude values.

Additionally, analyzing the diagrams in which the distributions are determined for six values of $\eta_{i}$ (Figs. 4, 6 and 8 ) it is noticeable that no matter whether a pulse with the value $\eta_{i}$ forces vibration or not, the error with which the distributions are determined is of the same order of magnitude.

When a distribution including pulses with smaller amplitudes $\left(\Phi_{5}, \Phi_{6}\right)$ forces vibration of systems with strong damping and short pulse response (Figs. 6-9), it seems to be possible to apply the described method of analysis in technological solutions. This conclusion refers to distributions computed for both six and three amplitude values.

\section{Conclusions}

The methodology adopted in this paper allowed to demonstrate how strong is the impact of pulse amplitudes on the difference between the actual distribution of pulses that force vibration of a damped oscillator and the distributions determined from a single function representing motion of such system. Answering the questions posed in the Introduction it can be state that even small differences between amplitudes of pulses forcing vibration of the system cause the occurrence of differences between distributions determined from the function representing system vibration. The attempt to answer the second of the questions, referring to vibration forced by a distribution of pulses with significant differences between their amplitudes, allows to draw further conclusions. It is evident that the more precisely the set of the finite number of values $\left\{\eta_{1}, \eta_{2}, \ldots, \eta_{k}\right\}$ is defined, the smaller will be errors in the determined distribution. Further, it was confirmed that for systems with strong damping and short pulse response, the presented method of analysis of vibration can be applied with success to technological solutions. A kind of added value issuing from the analysis is the information that systems should be designed bearing in mind that amplitudes of the strongest hits cause the smallest differences between the distributions of pulses actually forcing the vibration and distributions determined from the waveform representing observed motion of the system.

\section{Acknowledgments}

This research was supported in part by PL-Grid Infrastructure.

The project described in this paper has been executed within the project No. 11.11.130.955 


\section{References}

[1] M. Jabłoński, A. Ozga, Acta Phys. Pol. A 118, 74 (2010).

[2] M. Jabłoński, A. Ozga, Distribution of random pulses acting on a vibrating system as a function of its motion, AGH University of Science and Technology Press, Kraków 2013.

[3] M. Jabłoński, A. Ozga, Arch. Acoust. 34, 601 (2009).

[4] M. Jabłoński, A. Ozga, Mechanics 29, 163 (2010).

[5] M. Jabłoński, A. Ozga, Acta Phys. Pol. A 121, A-174 (2012).

[6] A. Ozga, Arch. Acoust. 39, 645 (2014).

[7] J. W.Creswell, Research Design. Qualitative, Quantitative, and Mixed Methods Approach, Publishing House Jagiellonian University (2013).

[8] A. Brański, in: Acoustic Waves, Ed. M.G. Beghi, ch. 18, InTech, Rijeka 2011, p. 397.

[9] M.S. Kozień, Acta Phys. Pol. A 123, 1029 (2013).
[10] M. Wiciak, Acta Phys. Pol. A 121, A-142 (2012).

[11] M. Wiciak, R. Trojanowski Acta Phys. Pol. A 123, 1048 (2013).

[12] R. Trojanowski, J. Wiciak, Acta Phys. Pol. A 121, A-148 (2012).

[13] M. Jabłoński, A. Ozga, T. Korbiel, P. Pawlik, Acta Phys. Pol. A 119, 977 (2011).

[14] E. Rusiński, P. Moczko, D. Pietrusiak, G. Przybyłek, Stroj. Vestn-J. MECH E 59, 556 (2013).

[15] J. Czmochowski, P. Moczko, P. Odyjas, D. Pietrusiak, Maintenance and Reliability 16, 211 (2014).

[18] M. Sibielak, J. Konieczny, J. Kowal, W. Raczka, D. Marszalik J Low Freq. Noise V A 32, 99 (2013).

[19] J. Konieczny, J. Kowal, W. Raczka, M. Sibielak, $J$ Low Freq. Noise V A 32, 81 (2013).

[16] A. Ozga, Acta Phys. Pol. A 123, 1034 (2013).

[17] A. Ozga, Acta Phys. Pol. A 125, A-159 (2014). 